\title{
Tethered spinal cord following repair of myelomeningocele
}

\author{
Roger J. Hudgins, M.D., and C. Lynn Gilreath, R.N. \\ Children's Healthcare of Atlanta, Atlanta, Georgia
}

\begin{abstract}
Object. The goal of this paper is to elucidate the clinical presentation of tethered cord syndrome (TCS) following repair of a myelomeningocele.

Methods. Approximately 10 to $30 \%$ of children will develop TCS following repair of a myelomeningocele. Because essentially all children with repaired myelomeningocele will have a tethered spinal cord, as demonstrated on MR imaging, the diagnosis of TCS is made based on clinical criteria. The six common clinical presentations of TCS are increased weakness $(55 \%)$, worsening gait $(54 \%)$, scoliosis $(51 \%)$, pain $(32 \%)$, orthopedic deformity $(11 \%)$, and urological dysfunction $(6 \%)$. The primary goal of surgery is to detach the spinal cord where it is adherent to the thecal sac, relieving the stretch on the terminal portion of the cord.

Conclusions. Early diagnosis and surgical release of the tethered cord results in stabilization or improvement in most cases. Because TCS may present with orthopedic and/or urological signs or symptoms, children with myelomeningocele should by followed, ideally in a multidisciplinary clinic, by neurosurgeons, orthopedic surgeons, and urologists who are aware of this condition.
\end{abstract}

\section{KEY WORDS • tethered spinal cord • myelomeningocele $・$ scoliosis}

All children in whom a myelomeningocele has been repaired will have a low-lying spinal cord that has at least some adhesions to the surrounding dura. This is caused by the inherent difficulty of closing the dura around the neural placode in an infant in such a way that the placode is bathed in CSF and not touching the dura. When contact occurs, scarring secures the spinal cord to the inside wall of the dura. At this time, there is no reliable technique that can prevent spinal cord tethering following repair of a myelomeningocele. ${ }^{14}$

Despite this, only approximately 10 to $30 \%$ of children with repaired myelomeningocele will develop neurological deterioration related to the tethered spinal cord. ${ }^{3}$ Thus, it is important for the neurosurgeon to understand that the presence of a low-lying tethered cord demonstrated on MR imaging does not by itself dictate intervention. Serial neurological, orthopedic, and urological examinations are imperative to detect gradual deterioration of spinal cord function so that intervention may avoid permanent loss of function.

The pathophysiology of TCS is discussed in this issue of Neurosurgical Focus by Yamada and colleagues ${ }^{12}$ and will only briefly be touched on here. This report will cover the clinical presentation, radiographic imaging, surgical treatment, and outcome of the child with TCS following closure of a myelomeningocele.

\footnotetext{
Abbreviations used in this paper: $\mathrm{CSF}=$ cerebrospinal fluid; $\mathrm{CT}=$ computerized tomography; $\mathrm{MR}=$ magnetic resonance; TCS $=$ tethered cord syndrome.
}

\section{CLINICAL PRESENTATION}

Most children with TCS following closure of a myelomeningocele present between 2 and 8 years of age, with a much smaller grouping between the ages of 10 and 12 years. ${ }^{5}$ There is an equal sex distribution.

There are six major presenting signs and symptoms in children with TCS: increased weakness (55\%), worsening gait $(54 \%)$, scoliosis $(51 \%)$, pain (32\%), orthopedic deformities $(11 \%)$, and decreased urological function $(6 \%){ }^{5}$ Although the ability to quantify strength will vary with the child's age and ability to cooperate, an attempt should be made by a trained neurological observer to document strength in the major muscle groups of all extremities. In a child with a repaired myelomeningocele, progressive weakness of one or both lower extremities is most likely caused by a tethered spinal cord. A change in gait requiring greater support to ambulate may be caused by weakness and/or increased spasticity.

It has now been well established by the work of McLone, et al., ${ }^{6}$ Reigel, et al., ${ }^{8}$ and others that TCS is a cause of scoliosis. The scoliosis occurs above the repair site and is not associated with vertebral anomalies. The highest incidence of scoliosis is seen in children with anomalies at thoracic levels (77\%). Reigel and coworkers ${ }^{8}$ have suggested that in some cases progressive lordosis and kyphosis may be improved by tethered cord release. These spinal deformities can lead to loss of sitting ability, development of chronic skin breakdown, and make ambulation more difficult. 
Pain as the main presenting symptom is more common in older children, at least in part because of their better ability to articulate discomfort. Back pain is most common, but there may also be a radicular component. Orthopedic deformities are usually due to imbalanced neural input to lower-extremity muscle groups. Commonly seen are hip dislocations, hammertoes, and high-arched feet. Bladder dysfunction is present in nearly $100 \%$ of children with a myelomeningocele; thus the urological presentation of TCS would be a change in voiding pattern. A common complaint is leakage of urine between catherizations when the child had previously been continent.

\section{RADIOGRAPHIC IMAGING}

In the child with myelomeningocele, all of the signs and symptoms listed previously can be caused by a shunt malfunction, even in the absence of the more classic symptoms of shunt blockage (headaches, vomiting, somnolence); thus, the first step in the radiographic evaluation of the child with suspected TCS is to obtain a CT scan of the brain. Enlarged ventricles from the child's baseline state mandate a shunt revision prior to release of the tethered cord. If the CT scan is equivocal, further evaluation of the shunt (shunt tap, radioisotope shunt function study, shunt exploration) may be necessary.

After adequate shunt function has been assured, an MR image of the spine is the study of choice (Fig. 1). It provides the neurosurgeon with a detailed road map of the distorted anatomy in both axial and sagittal planes. It will also allow, in some cases, for the preoperative identification of associated pathological entities such as dermoids, lipomas, syringomyelia, and diastematomyelia., ${ }^{5,11}$

Authors of several studies have discussed the use of imaging techniques to determine the presence of TCS. Vernet, et al., ${ }^{11}$ reported on the use of prone positioning during MR imaging in an attempt to see if anterior movement of the spinal cord would rule out TCS. They concluded that prone positioning was of no additional value in the evaluation of spinal cord tethering. Brezner and $\mathrm{Kay}^{3}$ used ultrasound to assess spinal cord motion and did not find a relationship between absence of motion and the presence of symptomatic TCS. It awaits further study to determine if MR imaging techniques such as diffusion imaging or spectroscopy of the lower spinal cord will be of value in the diagnosis of TCS. At this time the diagnosis of TCS is made by clinical criteria, with MR imaging serving to define the area of tethering and to reveal associated pathological entities.

\section{SURGICAL TECHNIQUE}

In children who present with TCS following myelomeningocele repair, the spinal cord is adherent to the the$\mathrm{cal} \mathrm{sac}$ at the site of the initial closure. Once tethered, normal movements of the spinal cord that would occur with flexion and extension of the spine as well as axial growth are restricted. This causes stretching of the lower aspect of the spinal cord and resultant injury. Yamada, et al., ${ }^{13}$ have demonstrated in an animal model that this abnormal stretch causes ischemia and mitochondrial anoxia. This type of vascular injury is consistent with the slow progression usually seen with TCS.
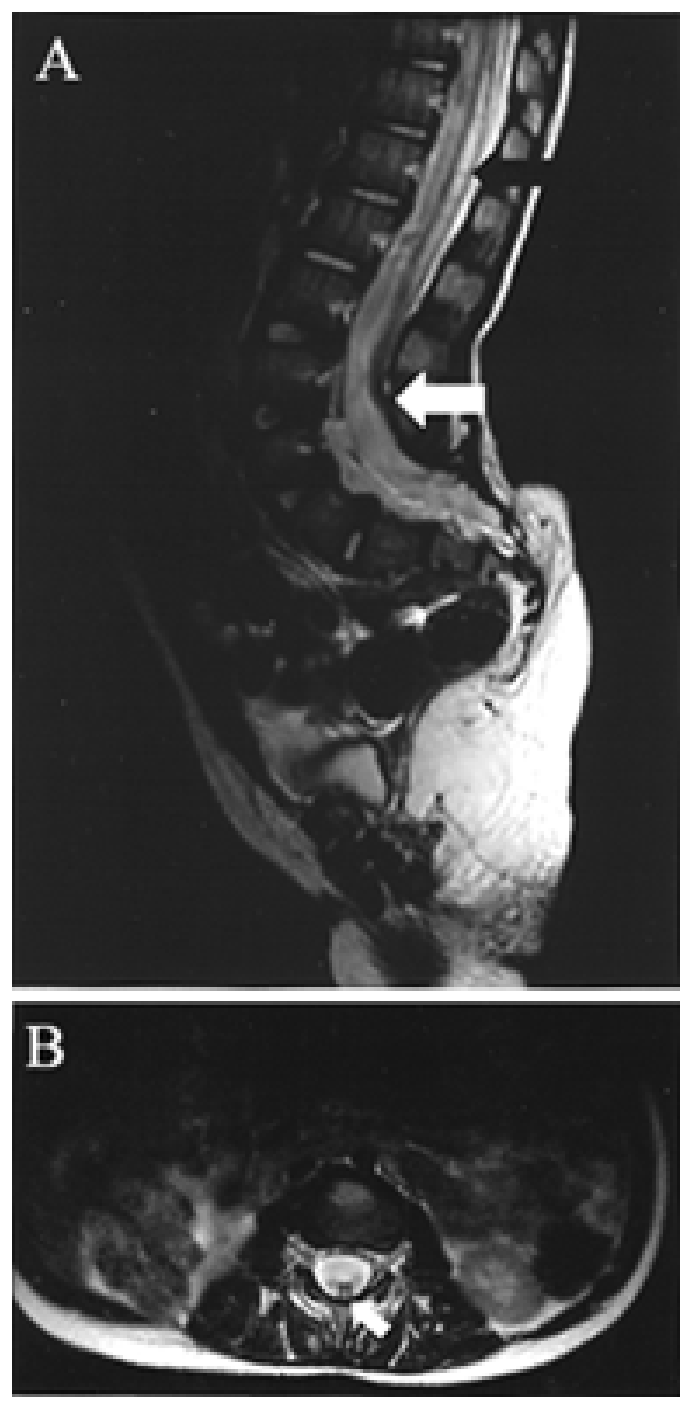

Fig. 1. A: Lumbosacral, midsagittal $\mathrm{T}_{2}$-weighted MR image demonstrating low-lying tethered spinal cord in a 6-year-old child with a repaired myelomeningocele. The spinal cord is displaced toward the posterior thecal sac and adherent to the dura at L-5 (white arrow). Hydromyelia is present in the lower spinal cord (black arrow). B: Axial $\mathrm{T}_{2}$-weighted MR image at the L-5 level of the same child, revealing tethering of the posterior spinal cord to the underside of the dura (arrow). Hydromyelia is again noted.

With this understanding of the pathophysiology of TCS, it is clear that the primary goal of surgical correction is to release the spinal cord from the adhering scar. The most common location for the spinal cord to be tethered is the lumbosacral spine, at the area of the previous closure. The usual operative finding is dense scar tissue between the posterior aspect of the spinal cord (neural placode) and the overlying dura.

A midline incision is used, even if the original closure incision was transverse. This allows exposure of the lowest intact spinous process and lamina. Laminectomy at this level will expose previously unopened dura. The dural opening begins at this point and proceeds caudally. The dura is opened in the midline until this is prevented by scar tissue. Whereas some use the $\mathrm{CO}_{2}$ laser for this dis- 
section, ${ }^{6,14}$ it has been the senior author's preference to use microscissors. Magnification, either with loupes or the operating microscope, is essential. Once midline scar is encountered, the dural opening veers laterally on each side, leaving adherent dura on the neural tissue. At all times the dissection should remain above the dorsal nerve roots to avoid injury to neural tissue. Intraoperative neurophysiological monitoring may help distinguish bands of scar tissue from nerve roots. ${ }^{9}$ The dural opening proceeds caudally until the spinal cord is freed of adherent scar and falls into the anterior thecal sac. Bleeding that occurs during the dissection can usually be controlled by thrombinsoaked Gelfoam and micropatties. The bipolar cautery should be used sparingly and only on low current. Complete untethering may not be achieved if there is dense scar on the lateral aspect of the spinal cord or if short sacral nerve roots do not allow full mobilization of the spinal cord.

Because additional disease is found in $30 \%$ of cases, the operative field should be inspected and further intervention performed as necessary. ${ }^{5} \mathrm{~A}$ tight terminal filum is cut if present and dermoids are removed. Diastematomyelia should be addressed if it is tethering the spinal cord either by arachnoid bands or bone spurs. Hydromyelia is seen in $18 \%$ of patients with spina bifida and may need to be treated (shunt, terminal ventriculostomy) if symptomatic or large. ${ }^{5}$

The dura is closed with the twin goals of creating a capacious CSF space that circumferentially surrounds the newly released neural tissue and to provide a watertight seal to prevent CSF leakage. Use of a dural graft or expander (adjacent fascia, bovine pericardium, Sialistic, Gore-Tex, or Medpor) may be necessary if the primary dural closure appears too tight. ${ }^{2,14}$ Whether these materials will prevent or slow retethering is unknown.

Following closure of the dura, the repair should involve multiple layers of tissue including muscle, fascia, subcutaneous tissue, and skin. It may be necessary to make relaxing incisions in the fascia to facilitate closure. Postoperatively, the child is maintained in a prone, flat position for 48 hours.

\section{OUTCOME}

\section{Motor and Gait Dysfunction}

As reported by Herman, et al., ${ }^{5}$ improvement by at least one spinal level was documented in $67 \%$ of children with myelomeningocele following release of the tethered spinal cord. Some motor improvement was noted in $79 \%$, including some originally thought to have a stable motor examination. This raises the intriguing question of whether children who have radiographically documented tethering, but are only minimally symptomatic, might benefit from untethering. At this point, there has not been a prospective study that addresses this issue. An improvement in walking endurance and stance was found in $72 \%$ of patients. $^{5}$

\section{Progression of Scoliosis}

Herman, et al., ${ }^{5}$ noted correction by at least $7^{\circ}$ in $51 \%$ of patients with myelomeningocele. Early postoperative progression of the scoliosis was noted only in those chil- dren with pre-untethering curves greater than 50\%. Progression of scoliosis after 1 year was noted in $37 \%$.

Reigel, et al., ${ }^{8}$ reported the results of tethered spinal cord release in 262 patients with myelomeningocele. They found that patients with thoracic levels experienced little benefit following tethered cord release, whereas most patients with lumbar and sacral levels had improvement or stabilization of scoliosis. Tethered cord release had little effect on lordosis in patients with low lumbar and sacral levels, but favorably altered the magnitude and incidence of lordosis for those with thoracic and upper lumbar levels. They also found that tethered cord release decreased the incidence and severity of kyphosis.

\section{Orthopedic Presentation and Pain}

Orthopedic foot deformities and/or hip dislocation improve in greater than $50 \%$ of children following tethered cord release. Pain is the preoperative symptom that improves most frequently following tethered cord surgery, with complete relief being achieved in $90 \%$ of patients. ${ }^{5}$

\section{Urological Dysfunction}

Herman et al., ${ }^{5}$ reported on improvement of urinary control in $33 \%$ of patients following tethered cord release. Fone, et al., ${ }^{4}$ did not find consistent urological benefit with "secondary" spinal cord tethering such as TCS associated with myelomeningocele, although they did report improvement in children with correction of tethering from occult spinal dysraphism. The majority of children presenting with nonurological signs and/or symptoms of TCS are found to have preoperative urodynamic abnormalities that improve postoperatively. ${ }^{7}$

\section{COMPLICATIONS}

The most common postoperative complication directly related to the surgical procedure is CSF leak. ${ }^{5}$ In some cases this can be treated with reclosure of the superficial layers; however, it may require a more extensive surgery to repair the leak at the level of the dural closure. Wound infection with or without meningitis can occur.

Even in the most skilled hands, new neurological and urological deficits may be present following surgery. The neural tissue in a tethered, ischemic spinal cord is susceptible to injury with minor disturbances. The probability of neurological morbidity increases with second or third tethered cord releases. ${ }^{1}$ As discussed previously, scoliosis may continue to progress after tethered cord release.

In our experience, shunt malfunction occurs in approximately $20 \%$ of children following tethered cord release. The presumptive cause in most cases is "low" or "negative" pressure hydrocephalus. ${ }^{10}$ Drainage of subarachnoid CSF during surgery may decrease global intracranial pressure to a point below the opening pressure of the shunt valve. The child presents with headaches, vomiting, and increased ventricle size on postoperative CT compared with baseline CT scans. We perform a radioisotope shunt function study to confirm shunt patency. If flow through the shunt is normal, the child is treated initially by pumping the shunt. If this does not work, the shunt is externalized, drained at low pressures, and then reinternalized when the ventricles return to baseline and the child is clinically improved. 


\section{CONCLUSIONS}

Based on radiography, essentially every child with a repaired myelomeningocele has a tethered spinal cord, although only 10 to $30 \%$ will develop signs or symptoms. Accordingly, the diagnosis of TCS is made on clinical criteria. Early detection and release of the tethered cord can stabilize or reverse deterioration in most cases. The presentation of TCS may be subtle and involve nonneurological conditions such as scoliosis or change in bladder continence. The multidisciplinary spina bifida clinic is the ideal way to follow these children so that all specialists involved in their care can discuss changes and plan treatments in a timely fashion.

\section{References}

1. Albright AL, Pollack IF, Adelson PD, et al: Outcome data and analysis in pediatric neurosurgery. Neurosurgery 45:101-106, 1999

2. Aliredjo RP, de Vries J, Menovsky T, et al: The use of GoreTex membrane for adhesion prevention in tethered spinal cord surgery: technical case reports. Neurosurgery 44:674-678, 1999

3. Brezner A, Kay B: Spinal cord ultrasonography in children with myelomeningocele. Dev Med Child Neurol 41:450-455, 1999

4. Fone PD, Vapnek JM, Litwiller SE, et al: Urodynamic findings in the tethered spinal cord syndrome: does surgical release improve bladder function? J Urol 157:604-609, 1997

5. Herman JM, McLone DG, Storrs BB, et al: Analysis of 153 patients with myelomeningocele or spinal lipoma reoperated upon for a tethered cord. Presentation, management, and outcome. Pediatr Neurosurg 19:243-249, 1993
6. McLone DG, Herman JM, Gabrieli AP, et al: Tethered cord as a cause of scoliosis in children with a myelomeningocele. Pediatr Neurosurg 16:8-13, 1990/1991

7. Palmer LS, Richards I, Kaplan WE: Subclinical changes in bladder function in children presenting with nonurological symptoms of the tethered cord syndrome. J Urol 159:231-234, 1998

8. Reigel DH, Tchernoukha K, Bazmi B, et al: Change in spinal curvature following release of tethered spinal cord associated with spina bifida. Pediatr Neurosurg 20:30-42, 1994

9. Sala F, Krzan MJ, Deletis V: Intraoperative neurophysiological monitoring in pediatric neurosurgery: why, when, how? Childs Nerv Syst 18:264-287, 2002

10. Vassilyadi M, Farmer JP, Montes JL: Negative-pressure hydrocephalus. J Neurosurg 83:486-490, 1995

11. Vernet O, O'Gorman AM, Farmer JP, et al: Use of the prone position in the MRI evaluation of spinal cord retethering. Pediatr Neurosurg 25:286-294, 1996

12. Yamada S, Won DJ, Yamada SM: Pathophysiology of tethered cord syndrome: correlation with symptomatology. Neurosurg Focus 16 (2):Article 6, 2004

13. Yamada S, Zinke DE, Sanders D: Pathophysiology of "tethered cord syndrome." J Neurosurg 54:494-503, 1981

14. Zide B, Constantini S, Epstein FJ: Prevention of recurrent tethered spinal cord. Pediatr Neurosurg 22:111-114, 1995

Manuscript received November 15, 2003.

Accepted in final form January 13, 2004.

Address reprint requests to: Roger J. Hudgins, M.D., 5455 Meridian Mark Road, Suite 540, Atlanta, Georgia 30342. 